\title{
All-optical quantum fluid spin beam splitter
}

\author{
A. Askitopoulos, ${ }^{1, *}$ A.V. Nalitov,${ }^{1, \dagger}$ E.S. Sedov,${ }^{1,2}$ L. Pickup,${ }^{1}$ E.D. Cherotchenko, ${ }^{1}$ \\ Z. Hatzopoulos, ${ }^{3}$ P.G. Savvidis, ${ }^{3,4,5}$ A.V. Kavokin, ${ }^{1,6}$ and P.G. Lagoudakis ${ }^{1,7}$ \\ ${ }^{1}$ School of Physics and Astronomy, University of Southampton, Southampton, SO171BJ, United Kingdom \\ ${ }^{2}$ Department of Physics and Applied Mathematics, \\ Vladimir State University named after A. G. and N. G. Stoletovs, Gorky street 87, 600000 Vladimir, Russia \\ ${ }^{3}$ Microelectronics Research Group, IESL-FORTH, P.O. Box 1527, 71110 Heraklion, Crete, Greece \\ ${ }^{4}$ Department of Materials Science and Technology, University of Crete, Crete, Greece \\ ${ }^{5}$ ITMO University, St. Petersburg 197101, Russia \\ ${ }^{6}$ Spin Optics Laboratory, St-Petersburg State University, 1, Ulianovskaya, St-Peterbsurg, 198504, Russia \\ ${ }^{7}$ Skolkovo Institute of Science and Technology Novaya St., 100, Skolkovo 143025, Russian Federation
}

(Dated: March 27, 2018)

\begin{abstract}
We investigate the spin behaviour of the first excited state of a polariton condensate in an optical trap by means of polarisation resolved spectroscopy. The interplay between the repulsive polariton interactions and the gain saturation results in a non-trivial spontaneous switching between the two quasi-degenerate spatial modes of the polariton condensate. As a result the polarisation pattern of the emitted light dramatically changes. Successful harnessing of this effect can lead to a spindemultiplexing device for polariton based optical integrated circuits.
\end{abstract}

Integratable spintronic devices are key elements for the implementation of spintronic circuits and spin based quantum processors [1]. An important component of this architecture is a coherent spin beam splitter that can separate particles having different spins [2]. Since the first experimental demonstration of stimulated amplification and Bose-Einstein Condensation (BEC) in semiconductor microcavities (MCs), polaritons have emerged as a promising platform for spintronic devices. They feature robust spin readout, long range spin transport [3], electrical control of the condensate energy and spin [4-6] and room temperature operation [7]. Recent state of the art MCs exhibit polariton lifetimes of hundreds of picoseconds and close to millimetre ballistic propagation lengths [8], while electrical creation of a polariton condensate has also been achieved [9]. Moreover, polaritonic systems have been shown to feature a wide range of resonant spin switching and multi-stability regimes $[10,11]$, while latest advances have also demonstrated spin switching and bistability in the non-resonant optical pumping configuration [12-14]. These advantages have lead to extensive theoretical suggestions of polariton based spin circuits $[15,16]$ as well as the realization of polariton optical spin filters [17]. However, a configuration that would allow for the directional and spatial separation of the spin components of a polariton condensate is yet to be demonstrated

Coherent polariton circuits can be engineered by modifying the potential landscape of the microcavity through deep etching of the structure. In this way a number of individual components of circuits have been realized such as polariton wires and routers $[18,19]$. Another approach is to modify the potential landscape for polaritons by optical means through the strong excitonic interactions that dominate in the excitation region [20,21]. This method has the advantage of being actively tunable: different circuits can be created and tested on the same sample simply by modulating the spatial profile of the excitation [22]. This idea has led to the realization of different components of future all-optical polariton based circuits such as all-optical polariton transistors [23] and amplifiers [24], while the hybridization of etching techniques and optical control has also been realized [25].

Although MC structures in the resonant excitation and OPO regime have been proposed as the basis of spin switching devices $[10,11,26]$, the fine control of the excitation energy required for the switching operation hinders efficient applicability of these techniques. Nevertheless, optical control of the circular polarization of polariton emission has also been demonstrated under nonresonant optical injection in planar MCs $[12,13,17,27$, 28], where intricate spin patterns have been observed due to the TE-TM splitting of the cavity mode [3, 29, 30].

In this work we study the first excited state of a polariton condensate in an annular optical trap. For a perfectly symmetric potential we expect a two-fold degeneracy of the first excited state $\left(\Psi_{01}, \Psi_{10}\right)$, while for balanced population of the spin states the degeneracy is four-fold (Fig.1(a)). By introducing a small ellipticity in the spatial profile of the excitation we lift the mode degeneracy $\left(\Delta \mathrm{E}_{l}\right)$ of the trap and observe at threshold a $p$-orbital state. In this regime, we identify a density dependent mode switching behaviour, where the coherent wavefunction spontaneously switches its orientation between two orthogonal axes. We allow for a small ellipticity in the polarization of the pump that lifts the spin degeneracy $\left(\Delta \mathrm{E}_{z}\right)$ of the trap by inducing unequal spin populations. Examining the spin components of the condensate we find that they switch independently and that for a range of parameters the two spinor condensates are oriented perpendicularly to each other giving rise to a butterfly spin pattern. We describe the system 


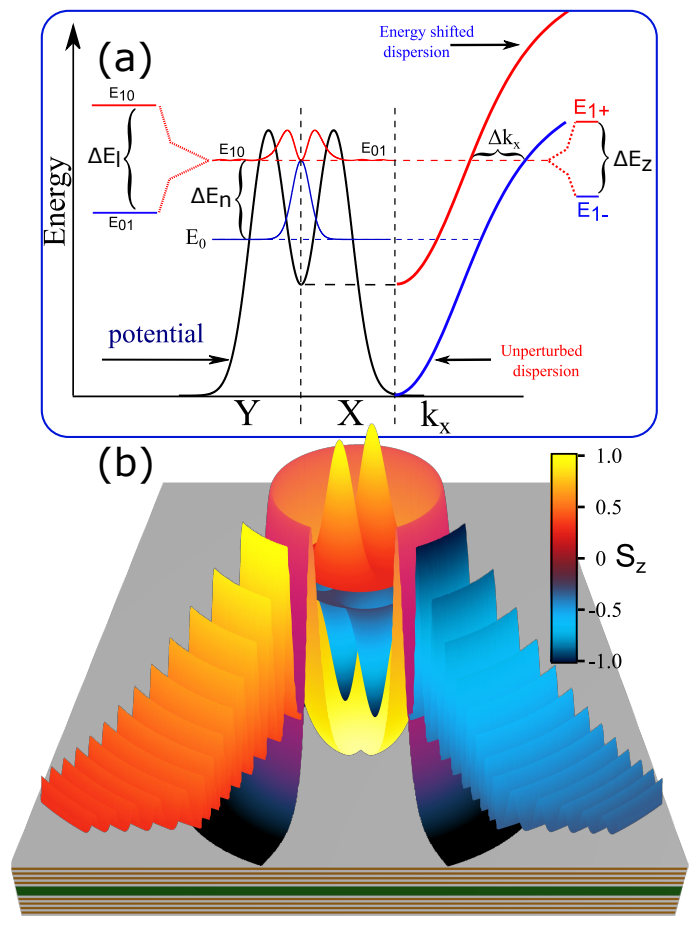

Figure 1. Schematic representation of: (a) the potential landscape of the system, detailing the four fold degeneracy and (b) spinor polariton condensate wavefunctions and tunnelling amplitudes in the trap.

behaviour within the framework of a two mode GrossPitaevskii equation (GPE) model, where the two modes are coupled due to the spatially inhomogeneous depletion of the exciton reservoir and the repulsive excitonpolariton interaction. Polaritons can escape the finite trapping potential through resonant tunnelling and are accelerated from the potential (by $\Delta k_{x}$ ). In the regime where the two spinor wavefunctions are oriented perpendicularly the system spatially separates polaritons escaping the trap based on their spin (Fig.1(b)). These results demonstrate that polariton condensates in optical traps offer unique possibilities for implementing spin logic and spin-demultiplexing operations in a solid state platform.

The structure we study here is a high quality $(Q=$ 16000) $5 \lambda / 2$ GaAs microcavity with four triplets of $10 \mathrm{~nm}$ GaAs quantum wells and a Rabi splitting of $\sim 9 \mathrm{meV}$. The sample is held in a cold finger continuous flow cryostat at $\sim 6 \mathrm{~K}$ and is excited with a single mode, continuous wave (CW) Ti:Sapphire source that is tuned to the minimum of the photonic stop band at $754 \mathrm{~nm}$. We use an acousto-optic modulator (AOM) with a frequency of $5 \mathrm{KHz}$ and a duty cycle of $1 \%$ to periodically switch off the CW optical excitation to avoid heating effects on the sample. We shape the spatial form of the excitation spot with the use of a phase spatial light modulator in Fourier space and project the laser onto the sample with an optical system of two lenses and a high numerical

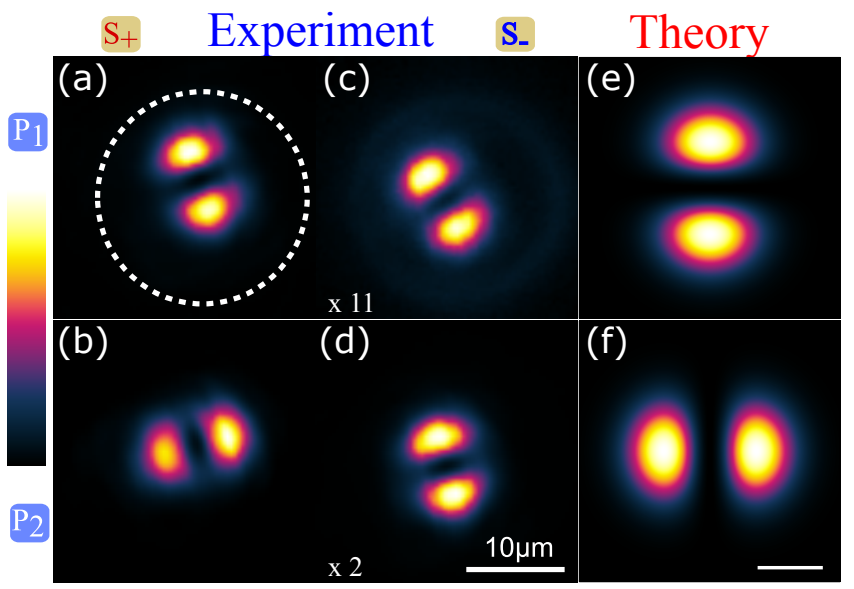

Figure 2. Orientation switching of single spin component. Normalized intensity maps of (a),(b) $S_{+}$spinor component for $P_{1}=1.2 P_{t h}$ and $P_{2}=1.4 P_{t h}$ and (c),(d) $S_{-}$spinor wavefunction. The white annulus in (a) outlines the excitation with the main axis of the ellipse along the vertical direction. (e-f) GPE simulation of a single component BEC for two densities above threshold reproducing the switching operation. The scale bar of (e) and (f) is in dimensionless units of $\sqrt{2 \beta / \alpha \Gamma}$.

aperture $(\mathrm{NA}=0.4)$ objective. The sample was excited at a negative detuning of $\Delta=-5 \mathrm{meV}$ were the lower polariton mode has a calculated exciton Hopfield coefficient of $X_{e}=0.25$, while the heavy hole exciton emission at $6 \mathrm{~K}$ is at $1.456 \mathrm{eV}$. Photoluminescence emission from the sample is collected through the same objective, while a dichroic mirror filters out the optical excitation and with the use of a $\lambda / 4$ wave-plate and a Wollaston prism we simultaneously project both spin components of the signal to our imaging apparatus.

We shape the non-resonant excitation into an annulus with radius $r_{\max }=10.8 \mu \mathrm{m}$ and a very small ellipticity $(\epsilon \sim 0.155)$, while the full width at half maximum (FWHM) of the laser profile is $\sim 2.5 \mu \mathrm{m}$. The annular excitation creates a nearly parabolic potential in the polariton energy landscape, effectively trapping polaritons and allowing condensation in the centre of the potential for a given excitation density threshold $\left(P_{t h} \approx 10.4 \pm 0.2 \mathrm{~mW}\right)$. For these conditions we observe that the dominant spinor state just above threshold $\left(P_{1}=1.2 P_{t h}\right)$ has a $p$-orbital symmetry that is oriented along the main axis of the ellipse as shown in Fig.2(a). Surprisingly, as we raise the excitation power slightly further $\left(P_{2}=1.4 P_{t h}\right)$ we observe a switching of the orientation of the $p$-orbital as shown in Fig.2(b) by $\pi / 2$. Here, the switching occurs between modes of the same order $\left(\Psi_{01}, \Psi_{10}\right)$ with an energy splitting of the order of $\sim 10 \mu \mathrm{eV}$ depending on the trap asymmetry, in contrast to switching between modes of different order where the energy spacing is significantly higher $\left(\Delta \mathrm{E}_{n} \sim 50 \mu \mathrm{eV}\right) \quad$ [31] and where the competition between modal gain and loss is enough to describe the transition $[32,33]$. Considering that the finite po- 

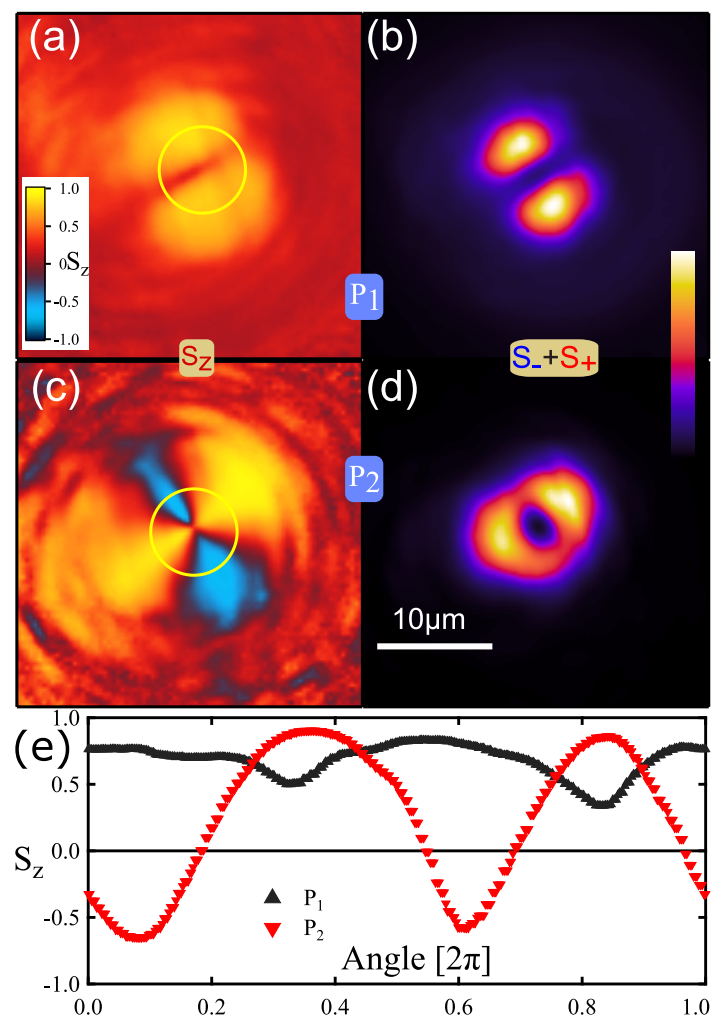

Figure 3. False colorscale spin textures and total intensity for: aligned spinor wave-functions at $P=P_{1}$ (a),(b) and for perpendicular spinor components at $P=P_{2}$ (c),(d) respectively. (e) $S_{z}$ component for $r=5 \mu \mathrm{m}$ radius, outlined by the yellow circle in (a) and (c), plotted against azimuthal angle for $P_{1}$ (black triangles) and for $P_{2}$ (inverse red triangles).

tential allows for polaritons to tunnel through the trap, this effect can in principle be used to seed and switch-on sub-threshold, optically or electrically created polariton states outside the optical trap and thus perform routing operations. [19, 23].

Polariton condensates confined in parabolic optical traps demonstrate remarkable spin effects such as spin switching [12, 13], spin bistability [14] and ferromagnetic phase transitions [12]. Polaritons have +1 and -1 spin projections to the growth axis of the $\mathrm{MC}$ that lead to right- and left-circular polarised photoluminescence. Resolving also the second spin component of the condensate, we note that at the onset of condensation both are in the same configuration, though one component is populated more strongly $(\sim \times 11)$, yielding a very high circular polarization of the emission of more than $80 \%$ Fig.2(a),(c). As we increase the density in order to reach the switching threshold, we observe that when the dominant component switches its orientation (Fig.2(b)), the low intensity spinor retains its initial configuration Fig.2(d). Nevertheless, as the two states are now primarily populated from different regions of the annular reservoir the relative density ratio is reduced to only $\sim 2.2$.

From the two spin images we construct the real space map of the third Stokes parameter $S_{z}=\left(S_{+}-S_{-}\right) /\left(S_{+}+\right.$ $\left.S_{-}\right)$, as well as the total intensity of the emission. In Figure 3(a),(b) $S_{z}$ and the total intensity are depicted for excitation power $P_{1}=1.2 P_{t h}$, showing the familiar spatial features of the $\Psi_{01}$ trapped state. For $P_{2}=1.4 P_{t h}$ the spatial profile of $S_{z}$ shows a spin precession around the core of the two orthogonally oriented components, whereas the total real space intensity distribution for this power resembles a doughnut or vortex state as expected from the linear superposition of the individual components 3(c),(d). In Figure 3(e) the angular profile of the polarization map for the two excitation powers across the yellow circle of Fig.3a,c is plotted, showing how the polarization profile of the collective mode changes from a nearly uniform angular distribution to an oscillating one.

Two-mode model. In the following we neglect the coupling between the two polariton spin components, assuming weak effective spin-orbit interaction and weak interaction of polaritons with opposite spins. We also assume slow spin relaxation in the exciton reservoir on the time-scale of its lifetime[34]. This allows us to treat each of the two decoupled spin components as an independent spin-less condensate. In the mean field approximation the latter is described by the wavefunction $\Psi(\mathbf{r}, t)$ which obeys the open-dissipative Gross-Pitaevskii equation (GPE) [35]:

$$
\mathrm{i} \frac{\partial \Psi}{\partial t}=\left[-\frac{\nabla^{2}}{2 m}+\frac{n}{2}(\alpha+\mathrm{i} \beta)+\frac{\alpha_{1}}{2}|\Psi|^{2}-\mathrm{i} \frac{\Gamma}{2}\right] \Psi .
$$

Here $m$ is the effective mass, $\Gamma$ is the inverse polariton lifetime, and $\hbar=1$. The effective complex potential for polaritons depends linearly on the exciton reservoir density $n(\mathbf{r}, t)$. Its real part stems from the polariton repulsion by the reservoir, the strength of which is given by $\alpha$. The imaginary part in turn describes stimulated scattering from the reservoir into the condensate, and is given by $\beta$. Finally, the repulsion strength of polaritons with the same spins is given by $\alpha_{1} \approx X^{2} \alpha$ with $X$ being the exciton Hopfield coefficient.

The GPE (1) is supplemented with the semi-classical equation on the exciton reservoir density:

$$
\frac{\partial n}{\partial t}=\mathrm{P}(\mathbf{r})-\left(\beta|\Psi|^{2}+\gamma\right) n,
$$

where the gain term $\mathrm{P}(\mathbf{r})$ is due to the inhomogeneous non-resonant optical pump and $\gamma$ is the reservoir decay rate, while we neglect exciton mobility in the reservoir.

To describe the polariton eigenstates confined in the elliptic optical trap, we assume a paraboloidal form of the reservoir density $n(r, \varphi)=n(\varphi) r^{2} / R^{2}$, where $R$ sets the size scale of the trap, and the angular density part

$$
n(\varphi)=N_{0}+n_{0}+n_{1} \cos (2 \varphi)+n_{2} \sin (2 \varphi),
$$


where $N_{0}$ is the reservoir density at the polariton lasing threshold, which is derived below, $n_{0}, n_{1}$, and $n_{2}$ are the angular harmonics of the reservoir density variation from $N_{0}$. Alternatively, the angular dependent part of the exciton reservoir density may be introduced as

$$
\delta n \cos (2(\varphi-\theta))=n_{1} \cos (2 \varphi)+n_{2} \sin (2 \varphi),
$$

with $\theta$ being the angle of the elliptic trap main axis.

In the linear regime the polaritons are thus confined in a complex harmonic potential

$$
U(r, \varphi)=\frac{m \omega_{+}^{2}}{2} r^{2} \cos (\varphi-\theta)^{2}+\frac{m \omega_{-}^{2}}{2} r^{2} \sin (\varphi-\theta)^{2},
$$

characterized with complex frequencies

$$
\omega_{ \pm}=\sqrt{\frac{N_{0} \alpha}{m R^{2}}}\left(1+\frac{\mathrm{i}}{2} \frac{\beta}{\alpha}\right)\left(1+\frac{n_{0} \pm \delta n}{2 N_{0}}\right),
$$

corresponding to the size quantisation along the two main axes of the ellipse. Here we assumed the realistic case $\alpha \gg \beta$ for simplicity. The eigenstate energies

$$
\begin{aligned}
E_{i, j}= & \sqrt{\frac{N_{0} \alpha}{m R^{2}}}\left(1+\frac{\mathrm{i}}{2} \frac{\beta}{\alpha}\right) \times \\
& {\left[\left(1+\frac{n_{0}}{2 N_{0}}\right)(1+i+j)+\frac{\delta n}{2 N_{0}}(i-j)\right], }
\end{aligned}
$$

are set by the two quantum numbers $i, j$, corresponding to size quantization along the elliptical axes. The wavefunctions, characterising the coherent polariton emission spatial profile, read:

$$
\Psi_{i, j}=\exp \left(-\frac{\xi_{+}^{2}+\xi_{-}^{2}}{2}\right) H_{i}\left(\xi_{+}\right) H_{j}\left(\xi_{-}\right),
$$

where $\xi_{+}=\sqrt{m \operatorname{Re}\left\{\omega_{+}\right\}} r \cos (\varphi-\theta)$ and $\xi_{-}=$ $\sqrt{m \operatorname{Re}\left\{\omega_{-}\right\}} r \sin (\varphi-\theta)$ and $H_{i}(x)$ are the Hermite polynomials.

To account for polariton tunnelling out of the trap potential one may add the following orders into the harmonic potential. Phenomenologically, the tunnelling rate may be accounted for in the state specific decay rate $\Gamma_{i, j}$ of the quantum state. We address the experimentally relevant case where the maximum gain-loss difference $\operatorname{Im}\left\{(1+2 i) \omega_{+}+(1+2 j) \omega_{-}\right\}-\Gamma_{i, j}$ corresponds to the first excited doublet of states $i=0, j=1$ and $i=1$, $j=0$. In the following we only keep the effective polariton decay rate $\Gamma=\Gamma_{0,1}=\Gamma_{1,0}$. The corresponding threshold reservoir density is then obtained by equating the polariton lasing gain term, given by the imaginary part of the confinement energy (7), to the loss term $\Gamma$ :

$$
N_{0}=\alpha m\left(\frac{\Gamma R}{2 \beta}\right)^{2}
$$

The doublet states (8) may be used to form a convenient basis of vortices, corresponding to a radially symmetric harmonic trap:

$$
\Psi_{ \pm}(r, \varphi)=\mathcal{A} r \exp \left( \pm \mathrm{i} \varphi-m \Gamma \frac{\alpha}{\beta} \frac{r^{2}}{2}\right)
$$

where $\mathcal{A}$ is the normalization constant. Low ellipticity of the trap allows us to neglect both ground and the higher excited states and treat it as a small perturbation, coupling the two basis states, in the vicinity of the polariton lasing threshold. We thus project the GPE (1) onto the basis (10), putting $\Psi=\psi_{+} \Psi_{+}+\psi_{-} \Psi_{-}$. Excluding the optical frequency we have in the rotating frame:

$$
\begin{aligned}
\mathrm{i} \frac{\mathrm{d} \psi_{ \pm}}{\mathrm{d} t}= & \frac{\Gamma}{2 N_{0}}\left(\frac{\alpha}{\beta}+\frac{\mathrm{i}}{2}\right)\left[n_{0} \psi_{ \pm}+\frac{\delta n}{2} e^{\mp 2 \mathrm{i} \theta} \psi_{\mp}\right] \\
& +\tilde{\alpha}_{1}\left[\left|\psi_{ \pm}\right|^{2} / 2+\left|\psi_{\mp}\right|^{2}\right] \psi_{ \pm},
\end{aligned}
$$

where $\tilde{\alpha}_{1}=\alpha_{1} \int\left|\Psi_{ \pm}\right|{ }^{4} \mathrm{~d}^{2} \mathbf{r}$. From Eq. (11) we derive the evolution of the condensate angular momentum $\mathbf{s}=$ $\psi^{\dagger} \boldsymbol{\sigma} \psi$, where $\psi=\left[\psi_{+} ; \psi_{-}\right]$and $\boldsymbol{\sigma}$ is the Pauli vector:

$$
\frac{\mathrm{d} \mathbf{s}}{\mathrm{d} t}=\frac{\Gamma}{2 N_{0}}\left[n_{0} \mathbf{s}+\frac{1}{2} \mathbf{n} s+\frac{\alpha}{\beta}[\mathbf{n} \times \mathbf{s}]\right]+\tilde{\alpha}_{1} s_{z}\left[\mathbf{e}_{z} \times \mathbf{s}\right]
$$

Here $\mathbf{n}=n_{1} \mathbf{e}_{x}+n_{2} \mathbf{e}_{y}$ with $n_{1}=\delta n \cos (2 \theta), n_{2}=$ $\delta n \sin (2 \theta)$, and $\mathbf{e}_{x}, \mathbf{e}_{y}, \mathbf{e}_{z}$ are the unitary vectors along the principal axes of the system.

As the typical reservoir decay rate $\gamma$ is much faster than the dynamics of the condensate, reservoir densities in Eq. (12) may be replaced with their equilibrium values, obtained from from Eq. (2) and linearized in $s$ : $n_{0}=(P-2 \tilde{\beta} s) / \gamma, n_{1}=\left(\delta P-2 \tilde{\beta} s_{x}\right) / \gamma, n_{2}=-2 \tilde{\beta} s_{y} / \gamma$. Here $P$ is the the angular independent part of the pumping power variation from the threshold $P_{0}=\gamma N_{0}, \delta P$ is its first angular harmonic and $\tilde{\beta}=\beta N_{0} \int\left|\Psi_{ \pm}\right|^{4} \mathrm{~d}^{2} \mathbf{r}$. Eq. (12) may be non-dimensionalized by scaling energies in $\gamma$ and densities in $N_{0}$. Defining for convenience $\mathbf{S}=2 \tilde{\beta} \mathbf{s}$, $\tau=\Gamma t / 2, \eta=\alpha / \beta$, and $\xi=\alpha_{1} /(\beta \Gamma)$ we arrive at:

$$
\begin{gathered}
\frac{\mathrm{d} \mathbf{S}}{\mathrm{d} \tau}=n_{0} \mathbf{S}+\frac{1}{2} \mathbf{n} S+\left[\left(\eta \mathbf{n}+\xi S_{z} \mathbf{e}_{z}\right) \times \mathbf{S}\right], \\
n_{0}=P-S, n_{1}=\delta P-S_{x}, n_{2}=-S_{y} .
\end{gathered}
$$

The first two terms in the right-hand part of Eq. (13) are the gain-loss part of the angular momentum evolution, while the third term describes its precession around an effective field, stemming from polariton repulsion from the reservoir and internal repulsion in the condensate.

We solve Eq. (13) with the following parameters: $\eta=10, \xi=20 \delta P=0.1$. There are two trivial stationary points of Eq. (13), characterized with condensate populations $S^{ \pm}(P)= \pm S_{x}^{ \pm}(P)=(2 P \pm \delta P) / 3$. While the lower populated branch $S_{-}$is unstable, the other one is destabilized by the non-linearities of Eq. (13) above the critical pumping power $P_{c}=0.2$ (corresponding to $\left.1.2 P_{t h}\right)$. At $P>P_{c}$ the only pair of stable stationary solutions $S(P)$ is symmetry breaking. Vorticity $\left(S_{z}\right)$ sign 

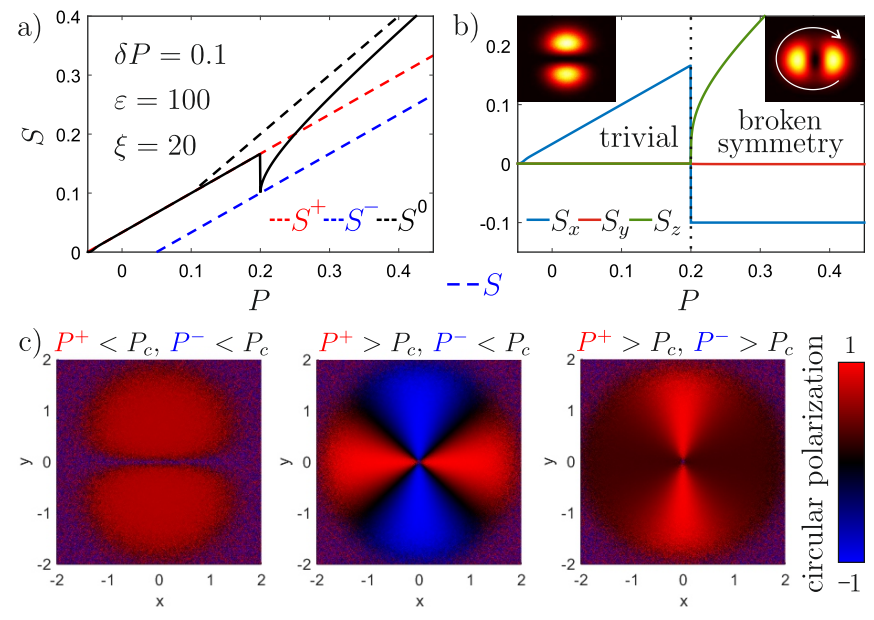

Figure 4. The results of the spinless polariton condensate model, stable solutions of Eq. (13). a) Population versus pumping power. Mode switching occurs at $P_{c}=0.2$. Trivial stationary branches $S^{ \pm}$and non-trivial branch for the photonic case $S^{0}$ are plotted with dashed lines. b) Angular momentum vectorial components. The spatial densities in the trivial and the broken symmetry phases are shown in the inset. c) Circular polarization patterns in decoupled spin components model. Butterfly spin pattern emerges where one of the circularly polarised components is above threshold, while the pumping of the other component is below threshold, e.g. $P^{+}>P_{c}$, while $P^{-}<P_{c}$.

in this phase is spontaneously chosen along with the sign of $S_{y}=-n_{0} S_{z} /(\eta \delta P)$. The sign of $S_{x}$ switches at the critical point, corresponding to the abrupt rotation of the condensate spatial density profile by $\pi / 2$ at the switching power $P_{c}$. We also consider for comparison the purely photonic case $\xi=0$ with no polariton repulsion. In the latter case the switching occurs at $P=0.1$ (corresponding to $\left.1.1 P_{t h}\right)$, the $S_{x}$ component holds its value above the critical pumping and the condensate occupation $S^{0}=P$ coincides with the asymptote of $S(P)$. The condensate population $S(P)$ is plotted in Fig. 4(a) along with the trivial branches $S^{ \pm}(P)$ and the vectorial components of $\mathbf{S}(P)$ are shown in Fig. 4(b).

The spin patterns emerge in the case of elliptical pumping, where the pumping in two spin polarisations $P^{ \pm}$are different. In the range of pumping powers where the predominantly pumped spin component of the condensate is in the broken symmetry phase $P^{+}>P_{c}$, while $P^{-}<P_{c}$, the two spin components are spatially separated, resulting in the butterfly spin pattern. Spin patterns corresponding to different pumping power ranges are shown in Fig. 4c. Note that artificial spatial noise is added in the regions where the condensate density is close to zero.

In conclusion, we have experimentally demonstrated and theoretically described a mode switching process in a polariton optical trap, where the condensate wavefunction spontaneously changes its orientation with an adiabatic pump power increase. The effect is described by the non-homogeneous depletion of the reservoir from dif- ferent spatial modes coupled with the nonlinear interactions of the system. This configuration can in principle be exploited for the design and implementation of on-chip polaritonic routers and transistors. By means of polarization resolved experiments, we have shown that the two spin components of a polariton condensate can switch their orientation independently. Further examination of this system can lead to the development of an integrable all-optical spin beam splitter or spin demultiplexer. Furthermore, as polariton condensate lattices have now been proposed as an architecture for implementing quantum simulators [36] also in the trap configuration [37], these $p$-orbital states can be used for unidirectional coupling between neighbouring nodes increasing the complexity of the systems that can be studied.

This work was carried out in the framework of the joint Russian-Greek project supported by Ministry of Education and Science of The Russian Federation (project RFMEFI61617X0085). A.N. acknowledges support from the Russian Foundation for Basic Research (Project No. 18-32-00434). The data from this paper can be obtained from the University of Southampton e-Prints research repository.

* correspondence address:Alexis.Askitopoulos@soton.ac.uk $\dagger$ correspondence address:A.Nalitov@soton.ac.uk

[1] I. Žutić, J. Fabian, and S. Das Sarma, Reviews of Modern Physics 76, 323 (2004).

[2] J. R. Petta, H. Lu, and A. C. Gossard, Science 327, 669 (2010).

[3] E. Kammann, T. C. H. Liew, H. Ohadi, P. Cilibrizzi, P. Tsotsis, Z. Hatzopoulos, P. G. Savvidis, A. V. Kavokin, and P. G. Lagoudakis, Physical Review Letters 109, 036404 (2012), 00079.

[4] G. Christmann, A. Askitopoulos, G. Deligeorgis, Z. Hatzopoulos, S. I. Tsintzos, P. G. Savvidis, and J. J. Baumberg, Applied Physics Letters 98, 081111 (2011).

[5] P. Tsotsis, S. Tsintzos, G. Christmann, P. Lagoudakis, O. Kyriienko, I. Shelykh, J. Baumberg, A. Kavokin, Z. Hatzopoulos, P. Eldridge, and P. Savvidis, Physical Review Applied 2, 014002 (2014).

[6] A. Dreismann, H. Ohadi, Y. del Valle-Inclan Redondo, R. Balili, Y. G. Rubo, S. I. Tsintzos, G. Deligeorgis, Z. Hatzopoulos, P. G. Savvidis, and J. J. Baumberg, Nature Materials 15, 1074 (2016).

[7] S. Christopoulos, G. B. H. von Högersthal, A. J. D. Grundy, P. G. Lagoudakis, A. V. Kavokin, J. J. Baumberg, G. Christmann, R. Butté, E. Feltin, J.-F. Carlin, and N. Grandjean, Physical Review Letters 98, 126405 (2007), 00709.

[8] B. Nelsen, G. Liu, M. Steger, D. W. Snoke, R. Balili, K. West, and L. Pfeiffer, Physical Review X 3, 041015 (2013), 00069.

[9] C. Schneider, A. Rahimi-Iman, N. Y. Kim, J. Fischer, I. G. Savenko, M. Amthor, M. Lermer, A. Wolf, L. Worschech, V. D. Kulakovskii, I. A. Shelykh, M. Kamp, S. Reitzenstein, A. Forchel, Y. Yamamoto, 
and S. Höfling, Nature 497, 348 (2013).

[10] A. Amo, T. C. H. Liew, C. Adrados, R. Houdré, E. Giacobino, A. V. Kavokin, and A. Bramati, Nature Photonics 4, 361 (2010).

[11] T. K. Paraïso, M. Wouters, Y. Léger, F. Morier-Genoud, and B. Deveaud-Plédran, Nature Materials 9, 655 (2010).

[12] H. Ohadi, A. Dreismann, Y. Rubo, F. Pinsker, Y. del Valle-Inclan Redondo, S. Tsintzos, Z. Hatzopoulos, P. Savvidis, and J. Baumberg, Physical Review X 5, 031002 (2015).

[13] A. Askitopoulos, K. Kalinin, T. C. H. Liew, P. Cilibrizzi, Z. Hatzopoulos, P. G. Savvidis, N. G. Berloff, and P. G. Lagoudakis, Physical Review B 93, 205307 (2016).

[14] L. Pickup, K. Kalinin, A. Askitopoulos, Z. Hatzopoulos, P. G. Savvidis, N. G. Berloff, and P. G. Lagoudakis, arXiv:1709.07351 [cond-mat] (2017), arXiv: 1709.07351.

[15] T. Espinosa-Ortega and T. C. H. Liew, Physical Review B 87, 195305 (2013).

[16] T. C. H. Liew, A. V. Kavokin, and I. A. Shelykh, Physical Review Letters 101, 016402 (2008).

[17] T. Gao, C. Antón, T. C. H. Liew, M. D. Martín, Z. Hatzopoulos, L. Viña, P. S. Eldridge, and P. G. Savvidis, Applied Physics Letters 107, 011106 (2015).

[18] E. Wertz, L. Ferrier, D. D. Solnyshkov, R. Johne, D. Sanvitto, A. Lemaître, I. Sagnes, R. Grousson, A. V. Kavokin, P. Senellart, G. Malpuech, and J. Bloch, Nature Physics 6, 860 (2010).

[19] F. Marsault, H. S. Nguyen, D. Tanese, A. Lemaître, E. Galopin, I. Sagnes, A. Amo, and J. Bloch, Applied Physics Letters 107, 201115 (2015).

[20] G. Tosi, G. Christmann, N. G. Berloff, P. Tsotsis, T. Gao, Z. Hatzopoulos, P. G. Savvidis, and J. J. Baumberg, Nature Physics 8, 190 (2012).

[21] M. Aßmann, F. Veit, M. Bayer, A. Löffler, S. Höfling, M. Kamp, and A. Forchel, Physical Review B 85, 155320 (2012).

[22] J. Schmutzler, P. Lewandowski, M. Aßmann, D. Niemietz, S. Schumacher, M. Kamp, C. Schneider, S. Höfling, and M. Bayer, Physical Review B 91, 195308 (2015).

[23] D. Ballarini, M. D. Giorgi, E. Cancellieri, R. Houdré, E. Giacobino, R. Cingolani, A. Bramati, G. Gigli, and D. Sanvitto, Nature Communications 4, ncomms2734 (2013).

[24] D. Niemietz, J. Schmutzler, P. Lewandowski, K. Winkler,
M. Aßmann, S. Schumacher, S. Brodbeck, M. Kamp, C. Schneider, S. Höfling, and M. Bayer, Physical Review B 93, 235301 (2016).

[25] C. Sturm, D. Tanese, H. S. Nguyen, H. Flayac, E. Galopin, A. Lemaître, I. Sagnes, D. Solnyshkov, A. Amo, G. Malpuech, and J. Bloch, Nature Communications 5, 3278 (2014).

[26] P. G. Lagoudakis, P. G. Savvidis, J. J. Baumberg, D. M. Whittaker, P. R. Eastham, M. S. Skolnick, and J. S. Roberts, Physical Review B 65, 161310 (2002).

[27] M. D. Martín, G. Aichmayr, L. Viña, and R. André, Physical Review Letters 89, 077402 (2002).

[28] C. Antón, S. Morina, T. Gao, P. S. Eldridge, T. C. H. Liew, M. D. Martín, Z. Hatzopoulos, P. G. Savvidis, I. A. Shelykh, and L. Viña, Physical Review B 91, 075305 (2015).

[29] P. Cilibrizzi, H. Sigurdsson, T. C. H. Liew, H. Ohadi, S. Wilkinson, A. Askitopoulos, I. A. Shelykh, and P. G. Lagoudakis, Physical Review B 92, 155308 (2015).

[30] P. Cilibrizzi, H. Sigurdsson, T. C. H. Liew, H. Ohadi, A. Askitopoulos, S. Brodbeck, C. Schneider, I. A. Shelykh, S. Höfling, J. Ruostekoski, and P. Lagoudakis, Physical Review B 94, 045315 (2016).

[31] A. Askitopoulos, T. C. H. Liew, H. Ohadi, Z. Hatzopoulos, P. G. Savvidis, and P. G. Lagoudakis, Physical Review B 92, 035305 (2015).

[32] Y. Sun, Y. Yoon, S. Khan, L. Ge, L. N. Pfeiffer, K. West, H. E. Tureci, D. W. Snoke, and K. A. Nelson, arXiv:1602.03024 [cond-mat, physics:quant-ph] (2016), arXiv: 1602.03024.

[33] L. Ge, A. Nersisyan, B. Oztop, and H. E. Tureci, arXiv:1311.4847 [cond-mat, physics:nlin] (2013), arXiv: 1311.4847.

[34] A. Vinattieri, J. Shah, T. C. Damen, D. S. Kim, L. N. Pfeiffer, M. Z. Maialle, and L. J. Sham, Physical Review B 50, 10868 (1994).

[35] M. Wouters and I. Carusotto, Physical Review Letters 99, 140402 (2007).

[36] N. G. Berloff, M. Silva, K. Kalinin, A. Askitopoulos, J. D. Töpfer, P. Cilibrizzi, W. Langbein, and P. G. Lagoudakis, Nature Materials 16, 1120 (2017).

[37] H. Ohadi, A. Ramsay, H. Sigurdsson, Y. del ValleInclan Redondo, S. Tsintzos, Z. Hatzopoulos, T. Liew, I. Shelykh, Y. Rubo, P. Savvidis, and J. Baumberg, Physical Review Letters 119, 067401 (2017). 